MITSUBISHI ELECTRIC RESEARCH LABORATORIES

http://www.merl.com

\title{
Novel multimode interference devices for wavelength beam splitting/combining
}

\author{
Kojima, K.; Koike-Akino, T.
}

TR2015-114 September 2015

\begin{abstract}
This paper reviews our recent device design activities on wavelength splitters/combiners. We start with blind optimization of MMIs having rectangular patches, and then based on the physical insight, the device structures are simplified to achieve even better performance. This methodology is applied to low insertion loss for two- and four-wavelength splitters/combiners, and it can be applied to other types of devices.
\end{abstract}

2015 Photonics in Switching

This work may not be copied or reproduced in whole or in part for any commercial purpose. Permission to copy in whole or in part without payment of fee is granted for nonprofit educational and research purposes provided that all such whole or partial copies include the following: a notice that such copying is by permission of Mitsubishi Electric Research Laboratories, Inc.; an acknowledgment of the authors and individual contributions to the work; and all applicable portions of the copyright notice. Copying, reproduction, or republishing for any other purpose shall require a license with payment of fee to Mitsubishi Electric Research Laboratories, Inc. All rights reserved. 



\title{
Novel Multimode Interference Devices for Wavelength Beam Splitting/Combining
}

\author{
Keisuke Kojima and Toshiaki Koike-Akino \\ Mitsubishi Electric Research Laboratories \\ Cambridge, MA 02139-1955, USA \\ Telephone: +1 (617) 621-7500 \\ Email: \{kojima, koike\}@merl.com
}

\begin{abstract}
This paper reviews our recent device design activities on wavelength splitters/combiners. We start with blind optimization of MMIs having rectangular patches, and then based on the physical insight, the device structures are simplified to achieve even better performance. This methodology is applied to low insertion loss for two- and four-wavelength splitters/combiners, and it can be applied to other types of devices.
\end{abstract}

\section{INTRODUCTION}

Recently, there have been increasing interest in designing optical devices using computer algorithms to optimize many parameters [1]-[4]. In these cases, device structures were optimized by using non-intuitive structures to outperform conventional straightforward structures.

InP-based photonic integrated circuits (PIC) have received much attention to monolithically integrate lasers, modulators, and wavelength combiners, to realize high performance and compact transmitters for wavelength division multiplexing optical communications. One of such applications is an optical Ethernet, where multiple wavelengths are combined into a single fiber [5].

A power combiner based on multi-mode interference (MMI) [6] has been used conventionally. Although its design and fabrication processes are well established, there is an inherent $3 N \mathrm{~dB}$ insertion loss for $2^{N} \times 1$ coupling $(N$ is a positive integer). Alternatively, $1 \times 2$ MMI-based wavelength splitters have been reported [7], whereas the device length needs to be long for narrow wavelength spacing.

An InP-based compact $4 \times 4$ arrayed waveguide grating (AWG) was fabricated [8] using deep reactive ion etching process. An InP-based $1 \times 2$ Mach-Zehnder interferometer (MZI) is another solution for wavelength coupler/splitter [9]. A compact $1 \times 4$ wavelength combiner/splitter has been demonstrated based on ring resonators on silicon-on-insulator platform [10]. However, this is not directly applicable to InGaAsP/InP material systems because very sharp bending is infeasible.

There are several approaches to shorten MMI couplers. For example, non-straight sidewall MMIs using binomial functions [11], exponential functions [12], and parabolic functions [13] have been studied to realize compact devices.

In this paper, we review a new design methodology using an optimization algorithm to design a large number of parameters at once. Then using the insight obtained from the structure, we simplify the device structure while further improving the device performances. These were applied to two- and fourwavelength splitters/combers. All of our simulations were conducted for InP-based wavelength combiners. However, the design methodology is readily applicable to other material systems, including silicon-on-insulator. Due to reciprocity, the transmittance characteristics of wavelength splitters are exactly the same as those of wavelength combiners.

\section{TWO-WAVELENGTH SPLITTER/COMBINER}

\section{A. Rectangular-patch devices}

We first designed a two-wavelength combiner with 14 small (length $<500 \mu \mathrm{m}$ ) patches using an optimizer [14]. The top view is shown in Fig. 1(a). The cross-sectional view is shown in Fig. 1(b), where thinner core layer regions give lower local effective refractive index.

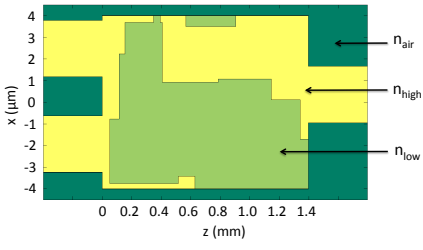

(a) Top view

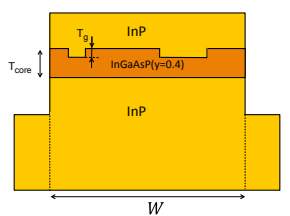

(b) Cross-section
Fig. 1: Structure of $2 \times 1$ wavelength combiner with 14 patches.

For the optimization, we used a metric of $\min \left(P_{1}^{\lambda_{1}}, P_{2}^{\lambda_{2}}\right)$, where $P_{1}^{\lambda_{1}}$ and $P_{2}^{\lambda_{2}}$ are the transmittances from the 1 st and 2nd input waveguide at wavelength $\lambda_{1}$ and $\lambda_{2}$, respectively. Transmittance is the ratio of the fundamental output mode power to the input fundamental mode power, calculated from the overlap integral including phase. This choice of metric means that we try to maximize the worst of the transmittance at two wavelengths. We randomly placed arbitrary sized 14 patches of low refractive index within the MMI. While fixing the MMI width to $8 \mu \mathrm{m}$, we optimized the length of MMI, widths and positions of input and output ports, and sizes and locations of the 14 patches. In order to optimize a total of 61 parameters simultaneously, we used finite-difference beam propagation method (FD-BPM) for very fast simulation, together with a covariance matrix adaptation evolutionary strategy (CMA-ES) [15]. As a result, we obtained the worst case transmittance of $83.8 \%$ (insertion loss of $0.77 \mathrm{~dB}$ ). 
We have also compared three widely-used global optimization methods, particle swarm optimization (PSO) [16] and continuous ant colony optimization (CACO) [17], as shown in Fig. 2. Even though CMA-ES did not converge very quickly, it almost always gave best performance at the end. It should also be stated that we also employed a "dithering" technique, in order to obtain more robust solutions.

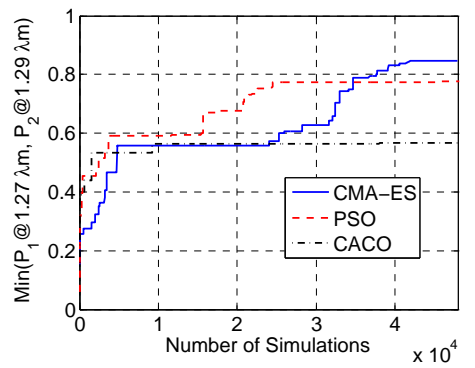

Fig. 2: Metric (worst of the transmittance at two wavelengths) as a function of number of simulations, for three widely-used global optimization algorithms, for $2 \times 1$ wavelength combiners with 14 patches.

The propagation patterns of the optimized device are shown in Fig. 3(a) and (b). These suggest that this device can be divided into three functional sections: a $2 \times 2$ coupler, two parallel waveguides, and a $2 \times 1$ coupler.

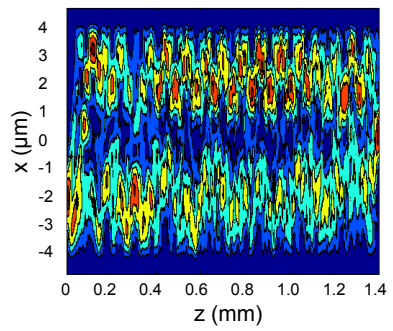

(a) $1.27 \mu \mathrm{m}$ input to port 1 .

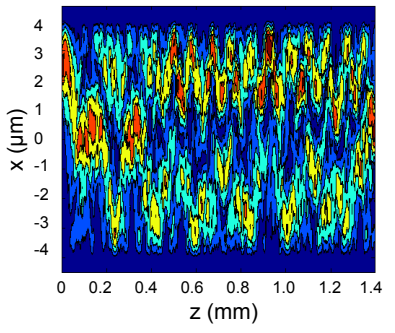

(b) $1.29 \mu \mathrm{m}$ input to port 2
Fig. 3: Propagation patterns of the input signals.

\section{B. Simplified structure}

Using the physical insight gained from the previous results, we devised a simplified device [18], [19]. The top view of the proposed device is shown in Fig. 4(a), where the lighter green part shows the lower refractive index region.

The cross-sectional view of the interferometer section is similar to Fig. 1(b), except that here there is a groove on one side. This creates nearly-localized propagation modes with distinct effective refractive indices in the MMI, unlike conventional uniform MMIs. Figures 4(b) and 4(c) show the lowest TE mode and the third lowest TE mode respectively, when the total MMI width is $W=6.0 \mu \mathrm{m}$, the patch width is $W_{1}=3.6 \mu \mathrm{m}$, core layer thickness is $T_{\text {core }}=0.5 \mu \mathrm{m}$, and groove thickness is $T_{\mathrm{g}}=0.2 \mu \mathrm{m}$. With CMA-ES, we optimized widths and offsets of input/output waveguides and the lengths of the $2 \times 2$ coupler, unbalanced interferometer,

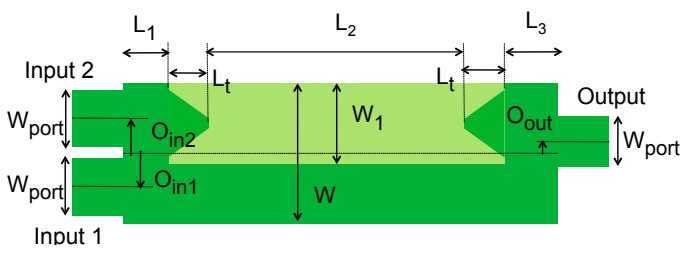

(a) Schematic view with dimensions

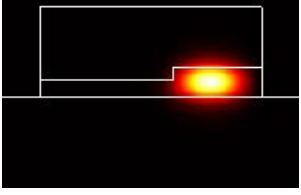

(b) The lowest TE mode

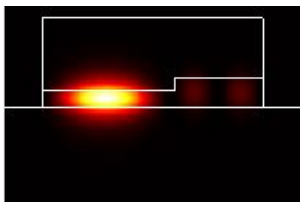

(c) The 3rd-lowest TE mode
Fig. 4: Simplified two beam combiner structure and modes at the MMI cross-section.

$2 \times 1$ coupler and taper sections simultaneously. The proposed device is simulated using the 3D simulation software FIMMWAVE. The total length of the device is $1272 \mu \mathrm{m}$.

Figures 5(a) and 5(b) show propagation patterns for $1.30 \mu \mathrm{m}$-wavelength input to port 1 , and $1.31 \mu \mathrm{m}$-wavelength input to port 2, respectively. In the interferometer section, two beams are confined into each section. However, since the two modes, as shown in Figs. 4(b) and 4(c), are not completely spatially separated, there are interference patterns as can be seen in the interferometer section. The wavelengthdependent transmittance for this device is shown in Fig. 5c. Since this device was optimized at $1.30 \mu \mathrm{m}$ and $1.31 \mu \mathrm{m}$, the transmittance (ratio of the output power to the input power) is as high as 0.870 (0.6 dB loss).

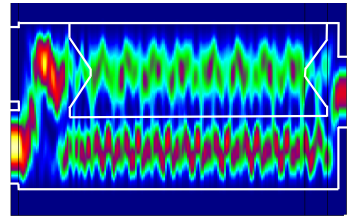

(a) $1.30 \mu \mathrm{m}$ input to port 1 .

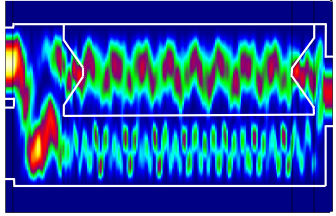

(b) $1.31 \mu \mathrm{m}$ input to port 2 .

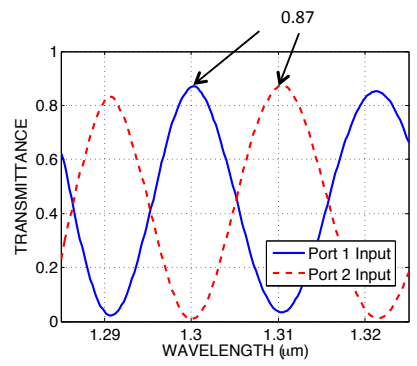

(c) Transmittance vs. wavelength.

Fig. 5: Propagation patterns and wavelength scan for a $1.30 / 1.31 \mu \mathrm{m}$ two-wavelength combiner with a simplified structure. 


\section{FOUR-WAVELENGTH SPLITTER/COMBINER}

\section{A. Rectangular-patch devices}

We extended the optimization strategy and designed a $4 \times 1$ wavelength combiner for $1271,1291,1311$, and $1331 \mathrm{~nm}$, using 16 patches [14]. In this case, a total of 75 parameters were optimized jointly. For this device, after using a global optimizer CMA-ES, the Nelder-Mead local optimizer [20] was used to fine tune the variables to gain extra $3 \%$ in transmittance. Figure 6(a) shows the optimized refractive index patterns, and Fig. 6(b) shows the transmittance from one of the 4 ports to the output port, as a function of wavelength. The MMI length was $1901 \mu \mathrm{m}$. The worst case insertion loss of $4.2 \mathrm{~dB}$ is achieved by simulation, which is a major improvement compared to the inherent $6.0 \mathrm{~dB}$ loss of a conventional MMI-based power combiner.

\section{B. Simplified structure}

Inspired by this rectangular-patch device, we proposed the use of a spline-curved index-step region in MMI as illustrated in Fig. 7(a) [21]. The device is simplified to have two regions separated by a spline curve, one of whose sides has an InGaAsP core thickness of $T_{\text {core }}$ and the other $T_{\mathrm{g}}$, as shown in Fig. 7(b), which depicts a cross-sectional view at the center of the MMI region. The one-sided width $W_{\mathrm{g}}$ is characterized by a smooth spline curve, which is a piece-wise cubic polynomial function obtained by a few control points.

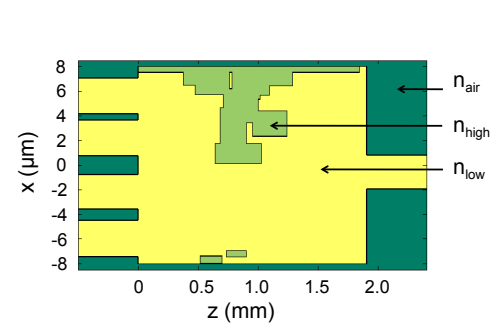

(a) Top view

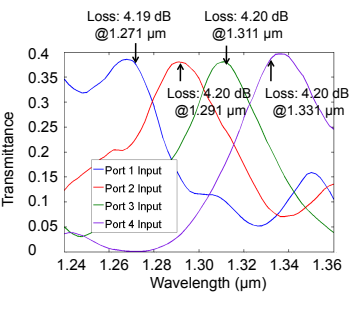

(b) Transmittance spectra
Fig. 6: Four-wavelength combiner with 16 patches.

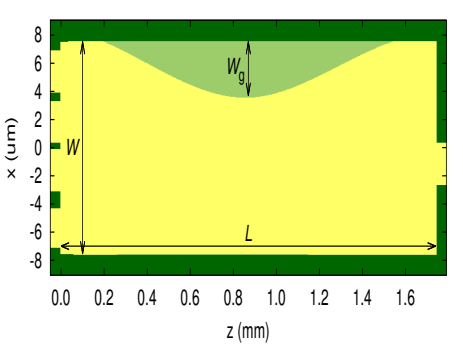

(a) Top view

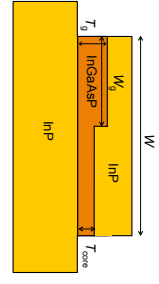

(b) Cross-sectional view
Fig. 7: Four-wavelength combiner with a spline curve.

We used 2D FD-BPM for beam propagation simulation, and CMA-ES was used with Nelder-Mead refinement. We optimized the position of spline control points, MMI width $W$, MMI length $L$, core thickness $T_{\text {core }}$ and $T_{\mathrm{g}}$ under reasonable boundary conditions. The total number of optimization parameters is at least 21 for a 3 -point spline case. We considered a
$4.5 \mathrm{~nm}$ wavelength spacing for the standard [5], whose precise wavelengths are $\lambda_{1}=1295.56, \lambda_{2}=1300.05, \lambda_{3}=1304.58$, and $\lambda_{4}=1309.14 \mathrm{~nm}$. The optimized device has length of $L=1.74 \mathrm{~mm}$, width of $W=15.1 \mu \mathrm{m}$ and core thicknesses of $T_{\text {core }}=0.25 \mu \mathrm{m}$ and $T_{\mathrm{g}}=0.53 \mu \mathrm{m}$. The spline curve has $W_{g}=3.96 \mu \mathrm{m}$ at the center of the MMI region. The insertion loss of the worst channel was $3.35 \mathrm{~dB}$, which was an improvement of $0.85 \mathrm{~dB}$ from the rectangular patch device, and a major improvement compared to the inherent $6.0 \mathrm{~dB}$ loss of a conventional MMI-based power combiner.

\section{OTHER APPLICATIONS}

Similar design methodology can be applied to other devices. We first optimized a polarization beam splitter with a single metal patch on an MMI. That beam propagation pattern inspired a polarization beam splitter based on a more concrete physical model [22].

\section{CONCLUSION}

This paper reviewed our recent device design activities on wavelength splitters/combiners. We start with blind optimization of sizes and positions of rectangular patches within MMIs with a metric designed to optimize under multiple criteria. The optimized structure revealed the underlining physical mechanism and we simplified the structure to achieve comparable or better performances. These design achieved low insertion loss for two- and four-wavelength splitters/combiners.

\section{ACKNOWLEDGMENT}

The authors would like to thank Bingnan Wang, Kieran Parsons, Selman Özbayat, Siddharth Singh, Eiji Yagyu, and Satoshi Nishikawa for their contributions and valuable comments on this work.

\section{REFERENCES}

[1] M. Spühler, et al., IEEE JLT 16 (1988) 1680-1685.

[2] T. P. Felici, D. F. G. Gallagher, Proc. SPIE 4986 (2003) 48-58.

[3] V. Liu, et al., Optics Expr. 20 (2012) 28388-28397.

[4] Y. Sakamaki, et al., IEEE JLT 25 (2007) 3511-3518.

[5] 100GBASE-LR4/ER4 in IEEE 802.3ba.

[6] S. Kanazawa, et al., IEEE Int'l Semiconductor Laser Conference (2010) $57-58$.

[7] C. Yao, et al., Opt. Expr. 20 (2012) 18248-18253.

[8] Y. Barbarin, et al., IEEE PTL 16 (2004) 2478-2480.

[9] K. Watanabe, et al., Electron. Lett. 47 (2011) 1245-1246.

[10] F. Xia, et al., InP and Related Materials Conf. (2006) 429-430.

[11] H.-C. Lu, W.-S. Wang, IEEE JLT 25 (2007) 2874-2878.

[12] J.-J. Wu, Progress in Electromagnetics Research C 1 (2008) 113-122.

[13] P. P. Sahu, Int'l Conf. on Design and Manufacturing 64 (2013) 215-223.

[14] S. Özbayat, et al., Opt. Commun. 322 (2014) 131-136.

[15] M. D. Gregory, et al., IEEE Trans. Antennas Propag., 59 (2011) 12751285.

[16] J. Robinson, Y. R. Samii, IEEE Trans. Antennas Propag., 52 (2004) 397-407.

[17] K. Socha, M. Dorigo, European J. Operational Res. 185 (2008) 1155 1173.

[18] S. Singh, et al., Opt. Expr. 22 (2014) 8533-8540.

[19] K. Kojima, et al., Integrated Photonics Research, Silicon and Nanophotonics (2014) JT4A.4.

[20] J. A. Nelder, R. Mead, Computer J. 7 (1965) 308-313.

[21] T. Koike-Akino, et al., Integrated Photonics Research, Silicon and Nanophotonics (2015) JM3A.16.

[22] K. Kojima, et al., Opt. Expr. 20 (2014) B371-B376. 